\title{
Unrepeatered transmission through ultra-long fiber laser cavities
}

\author{
J.D. Ania-Castañón ${ }^{1}$ and S.K. Turitsyn ${ }^{2}$ \\ ${ }^{1}$ Instituto de Óptica, CSIC, Serrano 121, Madrid 28006, Spain \\ ${ }^{2}$ Photonics Research Group, School of Engineering and Applied Science, Aston \\ University, Birmingham B47ET, United Kingdom \\ E-mail: juan.diego@,io.cfmac.csic.es
}

\begin{abstract}
We present a study on the potential use of ultra-long laser cavities for unrepeatered fibre communication, based on the theory of nonlinearity management. A comparison is offered between the performance of ultra-long lasers and standard bidirectional distributed amplification schemes in non-repeated transmission. Links based on both traditional (SMF/DCF) and modern Ultrawave transmission fibres are considered.
\end{abstract}

\section{Introduction}

Long-span unrepeatered WDM transmission systems can offer a significant cost reduction compared to in-line amplified links designed to operate at similar distances. Fibre links in the $250-500 \mathrm{~km}$ range represent a healthy fraction of the world's communications network and therefore, long systems without in-line amplification stages can be attractive solutions both in terms of maintenance and investment cost. Note that this kind of transmission systems is interesting not only for terrestrial links in difficult-to-access areas, but also in short-distance submarine applications. Several technologies have been proposed to enhance the performance of relatively long, unrepeatered links. These techniques include higher-order and bi-directional Raman amplification, remotely pumped EDFAs, as well as the use of advanced modulation formats and forward error-correction algorithms. [1-4]. Unrepeatered systems are rather challenging from the design viewpoint, as more input signal power is required than in conventional, periodically amplified schemes, making such systems inherently highly nonlinear. For this reason, the reduction of optical signal to noise ratio (OSNR) under the condition of limited nonlinear impairments, or vice versa suppression of the nonlinear effects under a fixed output OSNR is one of the key challenges in the design of unrepeatered systems. 
Ultra-long Raman active cavities have recently arisen as a potential efficient solution for providing broadband bi-directional, second-order Raman amplification with reduced amplified spontaneous emission (ASE) noise build-up [5-7]. In this paper we propose the idea of unrepeatered transmission links based on the implementation of an ultra-long laser cavity for the generation of an intermediate pumping wave which reduces signal power variation, with potential improvements in noise performance and reduced nonlinear distortion. We explore here the potential of using ultra-long, dispersionmanaged Raman lasers, as well as other second-order Raman amplification structures supported by fibre Bragg grating reflectors as non-repeated links. We perform a theoretical analysis, relying in the tried and tested theory of nonlinearity management [8-11] to compare the potential performance of an ultra-long laser based solution with that of a similarly complex (from the implementation point of view) 1st-order bidirectional distributed amplification scheme. Nonlinearity management theory has been shown to be a powerful tool for the optimization and comparative study of singlechannel and WDM system performance [9-11], in systems in which nonlinearity can be considered to contribute negatively to system performance. This approach greatly simplifies the analysis of systems when a large number of degrees of freedom are involved, and offers excellent results without the need to resort to direct NLSE transmission simulations.

1)
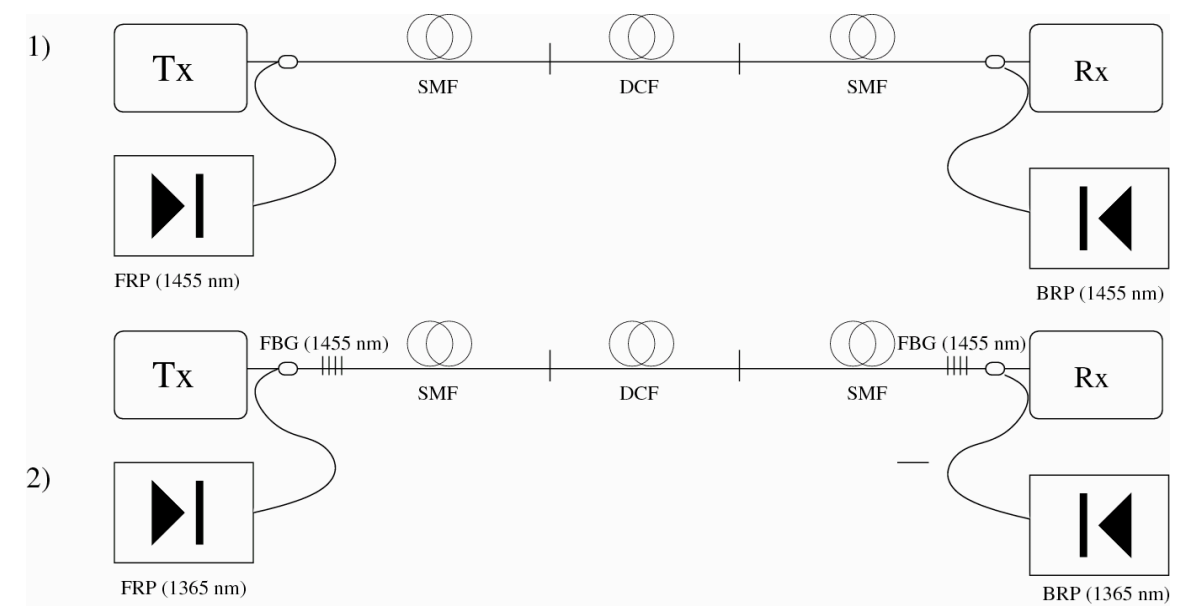

Fig. 1 The two configurations considered. Configuration 1 (top) uses simple bi-directional first-order pumping. Configuration 2 (bottom) is an ultra-long Raman laser link. 


\section{Model and system design}

As in the case of traditional transmission systems, the best performance of an unrepeatered link is found through the appropriate balance of noise and nonlinear effects. An interesting difference, though, is that, while in conventional systems optimisation is performed with the goal of achieving the maximum possible transmission distance, unrepeatered links must be optimised with the goal of achieving the best possible performance at a fixed distance. Although the principle might initially seem very similar, the rules to apply are different, and this, in particular, means that a proven amplification scheme for a $250 \mathrm{~km}$ reach might not be the best option for a 200 or $300 \mathrm{~km}$ link. This can be illustrated by monitoring the performance of different amplification configurations at different span lengths: Configuration 1 is just a standard bi-directionally pumped 1st-order Raman scheme, with identical pumps at both ends of the span at a wavelength of $1455 \mathrm{~nm}$. Configuration 2, on the other hand, is an ultralong Raman laser link using bidirectional pumps at $1365 \mathrm{~nm}$ and fibre Bragg gratings (FBGs) to transform the whole transmission span into a resonant cavity at $1455 \mathrm{~nm}$. Such a configuration, although relying only in two identical pumps, is equivalent to a 2nd-order bi-directionally pumped Raman scheme, as seen in [6]. The two configurations are depicted in figure 1.

We consider here dispersion-managed maps, with positive (PDF) and negative dispersion fibre (NDF) in a standard PDF-NDF-PDF configuration. For the sake of simplicity, but without loss of generality, we will focus in most of our examples on standard single-mode fibre (SMF) and dispersion-compensating fibre (DCF), although a performance comparison between such fibres and more modern UltraWave ${ }^{\mathrm{TM}}$ SLA (positive dispersion) and IDF (negative dispersion) fibres can be found below. Different span lengths are considered, varying from 100 to $300 \mathrm{~km}$ (i.e. from a total span loss of $26.5 \mathrm{~dB}$ to $83 \mathrm{~dB}$ ). The lengths of the positive and negative dispersion fibre sections are adjusted so that the total accumulated dispersion in the whole transmission link is zero. For our two sets of fibres this implies that $L_{D C F}=0.145 L_{S M F}$, and $L_{I D F}=0.333 L_{S L A}$ respectively, where $L$ indicates the total length for each type of fibre.

In order to compare the performance of the configurations under consideration from the point of view of nonlinearity management theory, it is convenient to resort to the numerical solution of the power balance equations, describing the evolution of the average power of each of different spectral components. 
These equations must account for all relevant contributions to noise, whether ASEgenerated or caused by Rayleigh backscattering $[12,5]$, in order to obtain an accurate estimation of the OSNR. Equations (1) to (5) below describe average power evolution of the spectral components on a system with two levels of Raman cascading.

$$
\begin{gathered}
\frac{d P_{P 1}^{ \pm}}{d z}=\mp \alpha_{1} P_{P 1}^{ \pm} \mp g_{1} \frac{v_{1}}{v_{2}} P_{P 1}^{ \pm}\left(P_{P 2}^{+}+P_{P 2}^{-}+4 h v_{2} \Delta v_{2}\left(1+\frac{1}{e^{h\left(v_{1}-v_{2}\right) / K_{B} T}-1}\right)\right) \pm \varepsilon_{1} P_{P 1}^{\mp} \\
\frac{d P_{P 2}^{ \pm}}{d z}=\mp \alpha_{2} P_{P 2}^{ \pm} \pm g_{1}\left(P_{P 2}^{ \pm}+2 h v_{2} \Delta v_{2}\left(1+\frac{1}{e^{h\left(v_{1}-v_{2}\right) / K_{B} T}-1}\right)\right)\left(P_{P 1}^{+}+P_{P 1}^{-}\right) \\
\mp g_{2} \frac{v_{2}}{v_{S}} P_{P 2}^{ \pm}\left(P_{S}+N_{S}^{+}+N_{S}^{-}+4 h v_{S} \Delta v_{S}\left(1+\frac{1}{e^{h\left(v_{2}-v_{S}\right) / K_{B} T}-1}\right)\right) \pm \varepsilon_{2} P_{P 2}^{\mp} \\
\quad \frac{d P_{S}}{d z}=-\alpha_{S} P_{S}+g_{2} P_{S}\left(P_{P 2}^{+}+P_{P 2}^{-}\right) \\
\frac{d N_{S}^{+}}{d z}=-\alpha_{S} N_{S}^{+}+g_{2}\left(N_{S}^{+}+2 h v_{S} \Delta v_{S}\left(1+\frac{1}{e^{h\left(v_{2}-v_{S}\right) / K_{B} T}-1}\right)\right)\left(P_{P 2}^{+}+P_{P 2}^{-}\right)+\varepsilon_{S} N_{S}^{-} \\
\frac{d N_{S}^{-}}{d z}=\alpha_{S} N_{S}^{-}-g_{2}\left(N_{S}^{-}+2 h v_{S} \Delta v_{S}\left(1+\frac{1}{e^{h\left(v_{2}-v_{S}\right) / K_{B} T}-1}\right)\right)\left(P_{P 2}^{+}+P_{P 2}^{-}\right)-\varepsilon_{S}\left(P_{S}+N_{S}^{+}\right)
\end{gathered}
$$

Here, the $(+)$ and (-) superscripts represent forward and backward propagation respectively, the 1,2 , and $\mathrm{S}$ subscripts identify the higher-order pump (1365 $\mathrm{nm}$ component), first-order pump (1455 nm) and signal (in the vicinities of $1550 \mathrm{~nm}$ ) respectively, $P_{P i}$ are the pump powers, $P_{S}$ are the signal powers and $N_{S}$ are the noise powers at the frequency of the signal, the $v_{i}$ are the corresponding frequencies of the pumps and signal, the $\Delta v_{i}$ represent the effective bandwidths of the secondary pump and the signal, the $g_{i}$ are the corresponding Raman gain coefficients (divided by the effective area) for each of the Raman transitions, the $\alpha_{i}$ are the fiber attenuations at each respective frequency, $h$ is Plank's constant, $K_{B}$ is Boltzmann's constant, $T$ is the absolute temperature of the fiber and the $\varepsilon_{i}$ are the double Rayleigh scattering coefficients of the fiber at each particular frequency. Note that the $\Delta v_{i}$ are limited, in the case of the secondary pump, by the bandwidth of the fiber Bragg gratings, and in the 
case of the signal, by the bandwidth of the channel (or combined bandwidth of the channels). Given that the Raman gain coefficient around the Stokes peak is nearly constant over a bandwidth of $\sim 3 \mathrm{THz}$ in silica fiber, the above set of equations can be used to monitor the average power of a single channel or the combined power of a limited number of WDM channels without the need to introduce a wavelength dependence for $g_{i}$.

The boundary conditions for the system of equations (1) - (5) depend on the particular amplification scheme under consideration. For configuration 1, in which no higherorder pumping is used, they can be expressed as:

$$
P_{P 1}^{+}(0)=P_{P 1}^{-}(L)=0 ; P_{P 2}^{+}(0)=P_{P 2}^{-}(L)=P_{0} ; N_{S}^{+}(0)=N_{0} ; N_{S}^{-}(L)=0 ; P_{S}(0)=P_{I N}(6 \mathrm{a})
$$

where $P_{0}$ is the input pump power for each of the pumps and $P_{I N}$ and $N_{0}$ stand for input signal and noise power respectively. For configuration 2, in which the $1455 \mathrm{~nm}$ component is generated directly by Raman conversion from the $1365 \mathrm{~nm}$ pump in the cavity, the boundary conditions can be written as (see [5]):

$$
P_{P 1}^{+}(0)=P_{P 1}^{-}(L)=P_{0} ; P_{P 2}^{+}(0)=R_{1} P_{P 2}^{-}(0) ; P_{P 2}^{-}(L)=R_{2} P_{P 2}^{+}(L) ; N_{S}^{+}(0)=N_{0} ; N_{S}^{-}(L)=0 ; P_{S}(0)=P_{I N}
$$

By solving the above set of ordinary differential equations for each of the configurations under study, both signal power evolution and optical signal-to-noise ratio (OSNR) can be monitored along the whole transmission link.

Table 1 summarizes the typical values of the relevant fiber parameters used in our simulations for SMF/DCF and Ultrawave ${ }^{\mathrm{TM}}$ fibers.

\section{Results and discussion}

Figures 2-top and 2-bottom show the evolution of signal and noise for a $250 \mathrm{~km} \mathrm{link}$ with the two configurations, considering a symmetric split of the pump power between the forward and backward direction in both cases, as well as an identical average input signal power of $0 \mathrm{dBm}$ and and input OSNR of $50 \mathrm{~dB}$ over a $1 \mathrm{~nm}$ bandwidth. The depicted noise contains both ASE in the signal bandwidth and Double-Rayleigh 
backscattering (DRB) noise, which can be very important with the high pump powers required for amplification in such long spans. The dispersion map is identical in both cases, with the DCF sitting in the middle of the span.

Amplification is applied to fully restore signal power at the end of the span. In both cases, the output OSNR over a $1 \mathrm{~nm}$ bandwidth is practically identical, of approximately $26.7 \mathrm{~dB}$. Applying the usual nonlinearity management theory approach, the accumulated nonlinearities are monitored through the accumulated nonlinear phase shift, defined as NPS $=\int_{0}^{L} \gamma(z) P_{S}(z) d z . N P S$ are much lower for the case of the ultralong cavity $\left(\mathrm{NPS}_{1}=2.31, \mathrm{NPS}_{2}=1.80\right)$, which given the identical dispersion map and output OSNR suggests that, for any fixed transmission format, configuration 2 should have the performance edge in this particular case. This will not necessarily hold true for longer transmission distances or more stringent OSNR requirements. This difference in accumulated NPS is due to the reduced signal power variation offered by configuration 2, which limits maximum signal power during propagation when compared to configuration 1 .
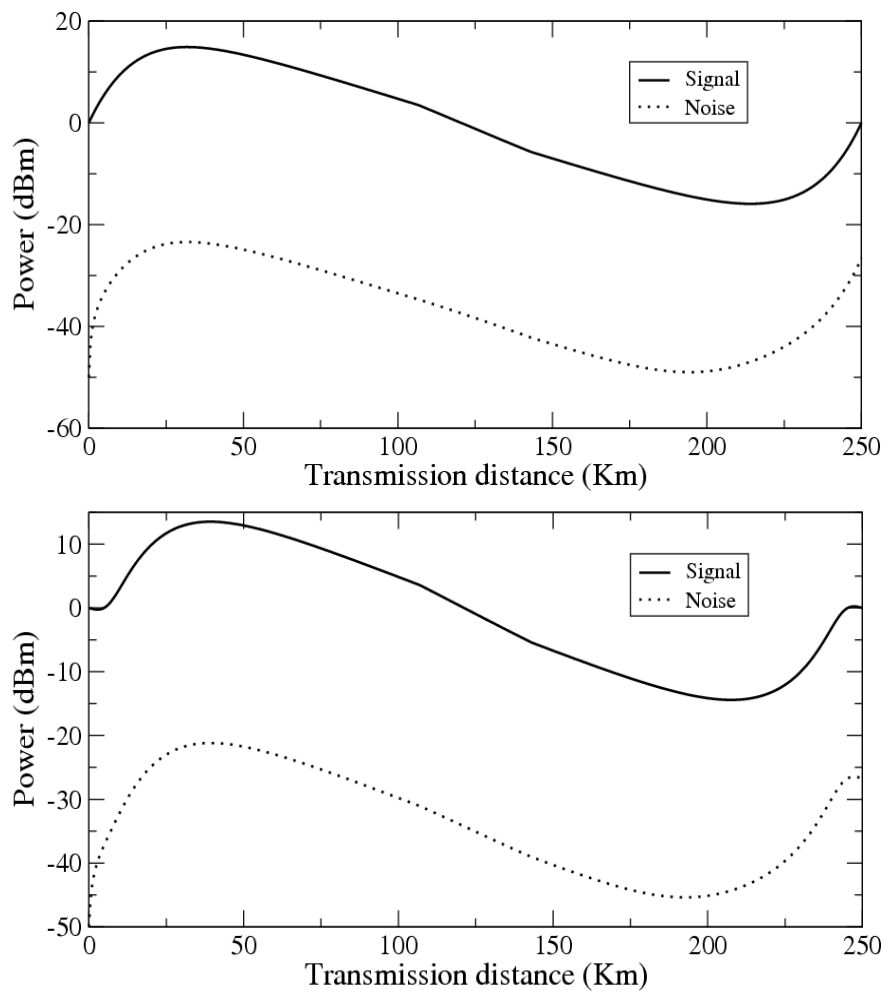

Fig. 2 Top - Signal and noise evolution over $250 \mathrm{~km}$ with a bi-directionally pumped Raman amplification scheme over SMF and DCF. Bottom - Signal and noise evolution over a $250 \mathrm{~km}$ ultra-long laser cavity. Both cases have the same input and output OSNRs, but the bottom case displays a $22 \%$ less NPS. 
Figure 3 illustrates the point by depicting the evolution of OSNR in both configurations for different input power levels. It can be seen that, at high input signal powers and long distances, the noise performance of ultra-long cavities quickly degrades beyond the barrier of $260 \mathrm{~km}$, becoming patently worse than that of configuration 1 . On the other hand, for low to moderate input powers ( $0 \mathrm{dBm}$ to $-5 \mathrm{dBm}$ average power), noise performance of configuration 2 is either superior or roughly equivalent to that of configuration 1, with the advantage of offering a lower impact of nonlinearities. At higher powers, on the other hand, configuration 1 has a slight advantage in terms of noise performance (while still being worse in terms of nonlinearity accumulation). Increasing signal power beyond a certain point has no positive effect whatsoever on the OSNR at long spans.

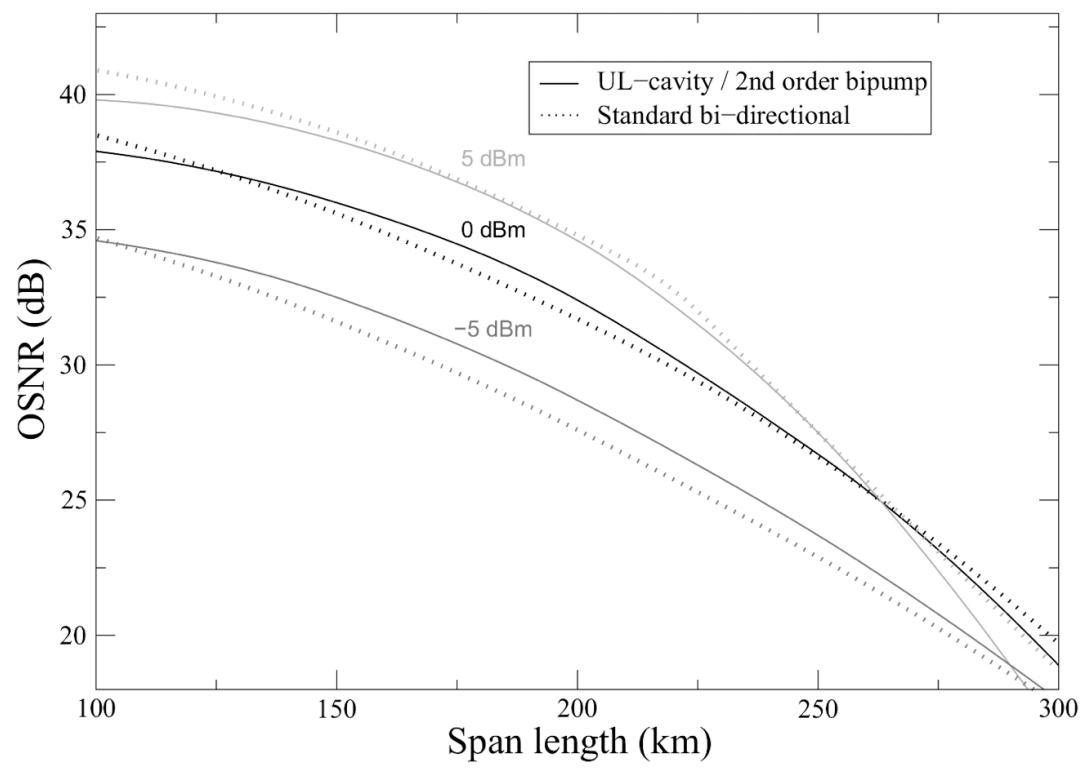

Fig. 3 OSNR for the SMF-DCF case at different input signal power levels and different transmission lengths.

At $260 \mathrm{~km}$, for both configurations, $0 \mathrm{dBm}$ and $5 \mathrm{dBm}$ average input signal power produce the same output OSNR. Indeed, for longer distances the OSNR drops sharply for the higher power case, due to pump depletion across the link and the increased incidence of double Rayleigh backscattering as input pump powers are increased. Note that for similar OSNRs in the same transmission map, lower input powers lead to reduced nonlinearities and hence a lower bit-error rate [11]. The results indicate that the 
best bit-error-rate for long spans with fully distributed amplification will usually be obtained by using moderate input signal powers.

Figure 4 displays the evolution of signal power excursion (defined as the difference between maximum and minimum power achieved along transmission, expressed in $\mathrm{dB}$ ) with increasing span length, for the sample case of a $0 \mathrm{dBm}$ average input signal power. The figure shows the results obtained both for standard SMF-DCF links and with systems using the more modern Ultrawave ${ }^{\mathrm{TM}}$ fibres, which display a larger effective area and matching dispersion slope. The curves show that the proposed scheme with ultra-long laser cavity is always superior to simple bi-directional pumping in terms of signal power excursion. This characteristic is important for the comparison of nonlinear impairments, as the accumulation of nonlinear effects is higher in the case of higher signal power excursion (i.e. higher power maxima during propagation), which is expected to impact deleteriously on the signal by increasing distortion. Note that when higher input signal powers are used, power excursion increases due to pump depletion. Cavities comprised of modern UltrawaveTM fibre present a lower power excursion than traditional SMF-DCF across the whole range of transmission lengths studied.

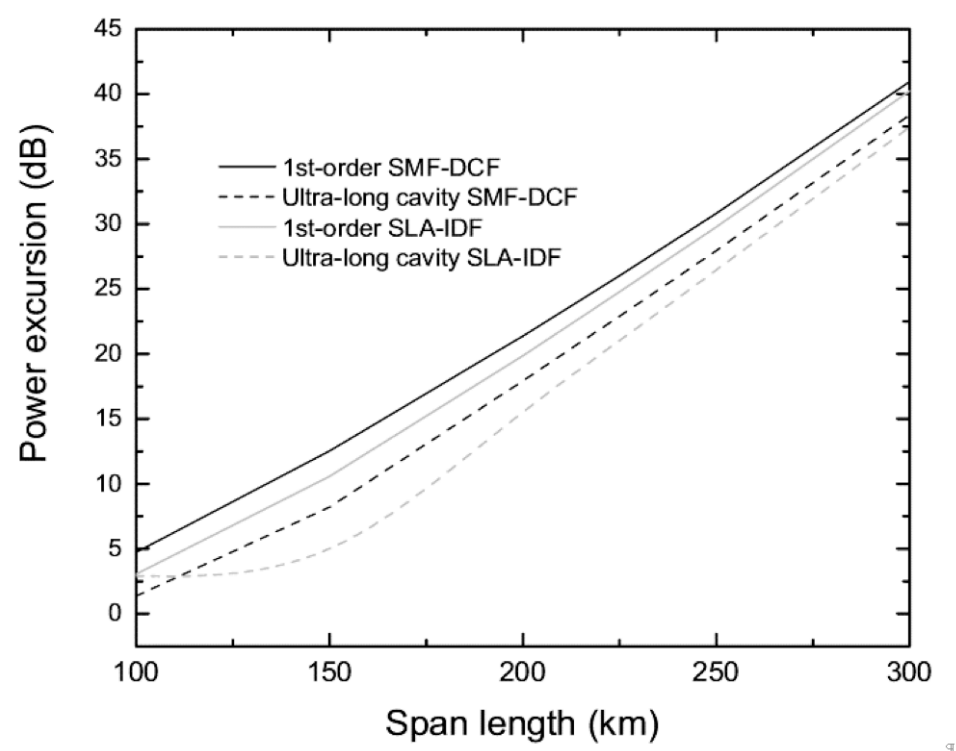

Fig. 4 Evolution of the signal power excursion with increasing span length, for the two schemes and two fibre configurations, with a $0 \mathrm{dBm}$ average input signal power.

\section{Conclusion}

While nonlinearity management is an adequate tool for the study and optimization of unrepeatered transmission systems, system design rules for this kind of transmission links are very different from the ones that apply to long-haul transmission, as the focus 
shifts from achieving the maximum possible transmission distance, to that of obtaining the best possible performance at a fixed distance. We have proposed, and studied from a nonlinearity management viewpoint, the application of higher-order distributed amplification schemes, based of the recently developed ultra-long Raman fiber laser architecture, to non-repeated fibre transmission. In standard transmission fibre links, for distances of up to $250 \mathrm{~km}$, the reduced power excursion and accumulated nonlinearities, as well as the slight improvement in OSNR, suggest that ultra-long cavity schemes could potentially offer a better overall performance than standard bi-directionally Raman amplified spans. The use of modern Ultrawave fibres leads to further improvement of the projected performance.

The authors would like to acknowledge the support of the EPSRC.

\section{References:}

[1] E. Brandon, P. Bousselet, I. Brylski, N. Tranvouez, D. A. Mongardien, "Bidirectional unrepeatered Transmission over $436 \mathrm{~km}$ using Third-order distributed Raman Amplification", in Proceedings of ECOC 2007 (IEEE, Berlin, Germany., 2007) paper 2.3.4

[2] P. Bousselet, D. A. Mongardien, P. Brindel, H. Bissessur, E. Dutisseuil, E. Brandon, I. Brylski, 485km Unrepeatered 4x43Gb/s NRZ-DPSK Transmission", in Proceedings of OFC 2008 (OSA, San Diego, USA, 2008) paper OMQ7

[3] B. Bakhshi, S. B. Papernyi, M. Manna, G. Mohs, P. Corbett, B. Shum-Tim, E.A. Golovchenko, "320 Gb/s (32 x $12.3 \mathrm{~Gb} / \mathrm{s}) 410 \mathrm{~km}$ repeaterless WDM system ready for field deployment", in Proceedings of ECOC 2005 (IEEE, Glasgow, 2005) paper Mo4.2.5

[4] T. Mizuochi, K. Kinjo, S. Kajiya, T. Tokura and K. Motoshima, "Bidirectional unrepeatered $43 \mathrm{Gbit} / \mathrm{s}$ WDM transmission with $\mathrm{C} / \mathrm{L}$ Band-Separated Raman amplification", J. Lightwave Technol. 20, 2079 (2002)

[5] J.D. Ania-Castañón, "Quasi-lossless transmission using second-order Raman amplification and fibre Bragg gratings", Opt. Express, 12, 4372 (2004)

[6] J.D. Ania-Castañón, T.J. Ellingham, R. Ibbotson, X.Chen, L. Zhang, S.K. Turitsyn, "Ultralong Raman fiber lasers as virtually lossless optical media", Phys. Rev. Lett., 96, $023902(2006)$ 
[7] T.J. Ellingham, J.D. Ania-Castañón, R. Ibbotson, X. Chen, L. Zhang, S.K. Turitsyn, "Quasi-Lossless Optical Links for Broad-Band Transmission and Data Processing", IEEE Photon. Technol. Lett. 18(1), 268 - 270 (2006)

[8] R. Gabitov, P. M. Lushnikov, "Nonlinearity management in a dispersion-managed system," Opt. Lett. 27, 113-115 (2002)

[9] J.D. Ania-Castañón, I.O. Nasieva, N. Kurukitkoson, S.K. Turitsyn, C. Borsier, E. Pincemin, "Nonlinearity management in fiber transmission systems with hybrid amplification" Opt. Commun. 233, 353-357 (2004)

[10] I.O. Nasieva, J.D. Ania-Castañón and S.K. Turitsyn, "Nonlinearity management in fibre links with distributed amplification," Electron. Lett. 39, 856-859 (2003)

[11] J. D. Ania-Castañón, S. K. Turitsyn, A. Tonello, S. Wabnitz, and E. Pincemin, "Multi-level optimization of a fiber transmission system via nonlinearity management", Optics Express, 14, 8065 (2006)

[12] J.D. Ania-Castañón and S.K. Turitsyn, "Noise and gain optimisation in bidirectionally pumped dispersion compensating amplifier modules", Opt. Commun. 224, 107 (2003)

\section{Tables:}

Table 1: Fiber parameters

\begin{tabular}{lllll}
\hline Parameter & SMF & DCF & SLA & IDF \\
$\alpha_{1}$ & $0.38 \mathrm{~dB} \mathrm{~km}^{-1}$ & $0.60 \mathrm{~dB} \mathrm{~km}^{-1}$ & $0.38 \mathrm{~dB} \mathrm{~km}^{-1}$ & $0.43 \mathrm{~dB} \mathrm{~km}^{-1}$ \\
$\alpha_{2}$ & $0.24 \mathrm{~dB} \mathrm{~km}^{-1}$ & $0.30 \mathrm{~dB} \mathrm{~km}^{-1}$ & $0.234 \mathrm{~dB} \mathrm{~km}^{-1}$ & $0.30 \mathrm{~dB} \mathrm{~km}^{-1}$ \\
$\alpha_{S}$ & $0.2 \mathrm{~dB} \mathrm{~km}^{-1}$ & $0.26 \mathrm{~dB} \mathrm{~km}^{-1}$ & $0.19 \mathrm{~dB} \mathrm{~km}^{-1}$ & $0.233 \mathrm{~dB} \mathrm{~km}^{-1}$ \\
$g_{1}$ & $0.52 \mathrm{~W}^{-1} \mathrm{~km}^{-1}$ & $2.06 \mathrm{~W}^{-1} \mathrm{~km}^{-1}$ & $0.36 \mathrm{~W}^{-1} \mathrm{~km}^{-1}$ & $0.52 \mathrm{~W}^{-1} \mathrm{~km}^{-1}$ \\
$g_{2}$ & $0.42 \mathrm{~W}^{-1} \mathrm{~km}^{-1}$ & $1.75 \mathrm{~W}^{-1} \mathrm{~km}^{-1}$ & $0.29 \mathrm{~W}^{-1} \mathrm{~km}^{-1}$ & $0.42 \mathrm{~W}^{-1} \mathrm{~km}^{-1}$ \\
$\varepsilon_{1}$ & $1.01 \times 10^{-4} \mathrm{~km}^{-1}$ & $1.1 \times 10^{-3} \mathrm{~km}^{-1}$ & $1.0 \times 10^{-4} \mathrm{~km}^{-1}$ & $4.0 \times 10^{-4} \mathrm{~km}^{-1}$ \\
$\varepsilon_{2}$ & $6 \times 10^{-5} \mathrm{~km}^{-1}$ & $7.1 \times 10^{-4} \mathrm{~km}^{-1}$ & $5.0 \times 10^{-5} \mathrm{~km}^{-1}$ & $2.2 \times 10^{-4} \mathrm{~km}^{-1}$ \\
$\varepsilon_{S}$ & $4.3 \times 10^{-5} \mathrm{~km}^{-1}$ & $5.1 \times 10^{-5} \mathrm{~km}^{-1}$ & $4.0 \times 10^{-5} \mathrm{~km}^{-1}$ & $1.6 \times 10^{-4} \mathrm{~km}^{-1}$ \\
\hline
\end{tabular}

